\title{
Fast Fourier-based DSP algorithm for auditory motion experiments
}

\author{
KOUROSH SABERI \\ University of California, Irvine, California
}

\begin{abstract}
A digital signal-processing (DSP) technique for rapid generation of complex auditory motion stimuli based on dynamic linear changes in interaural delay is described. In this technique, a pair of complementary discrete Fourier transforms (DFTs) for which the component spacing in one series is different than that of the other is used. The appeal of this technique is its wide applicability, since it can generate real-time motion stimuli of any velocity and starting interaural delay for complex broadband or filtered noise waveforms and nonstationary sounds such as speech, music, and other natural sounds.
\end{abstract}

The mechanisms that underlie auditory motion perception have been investigated for over a century (Dove, 1839; Peterson, 1916; Rayleigh, 1876; Thompson, 1877, 1878; Valentine, 1928). In the last few decades, motion detection has been examined in psychophysical studies as a function of dynamic variation in interaural cues (Blauert, 1972; Grantham, 1984; Grantham \& Wightman, 1978), the role of velocity (Altman \& Viskov, 1977; Grantham, 1986; Perrott \& Musicant, 1977a; Waugh, Strybel, \& Perrott, 1979), movement trajectory (Saberi \& Perrott, 1990), stimulus spectrum (Chandler \& Grantham, 1992; Perrott \& Tucker, 1988; Saberi, 1996), as well as other dynamic stimulus features (Grantham, 1986; Perrott \& Musicant, 1977b; Perrott \& Nelson, 1969; Strybel \& Fujimoto, 2000; Strybel, Manligas, \& Perrott, 1989; Strybel, Span, \& Witty, 1998). In physiological studies, brainstem and cortical neurons have been identified in a variety of species that respond preferentially to a specific direction of auditory-source movement but are nonresponsive to stationary sounds (Ahissar, Ahissar, Bergman, \& Vaadia., 1992; Moiseff \& Haresign, 1992; Rauschecker \& Harris, 1989; Spitzer \& Semple, 1991; Stumpf, Toronchuk, \& Cynader, 1992). The study of auditory motion continues to be a main topic of research in traditional areas of the hearing sciences (psychophysics and neurophysiology) and is rapidly expanding to new areas, such as human brain imaging. Recent human neuroimaging experiments (Pavani, Macaluso, Warren, Driver, \& Griffiths, 2002; Warren, Zielinski, Green, Rauschecker, \& Griffiths, 2002) have implicated the parietal lobe and planum temporale in auditory motion processing, although this finding remains in dispute (Smith, Okada, Saberi, \& Hickok, 2004).

Correspondence concerning this article should be addressed to K. Saberi, Department of Cognitive Sciences, University of California, 3151 Social Science Plaza, Irvine, CA 92697-5100 (e-mail: saberi@ uci.edu).
In spite of its continued importance, the study of auditory motion perception has been limited by a number of difficulties. For example, constructing real-moving soundsource hardware at accurate velocities either in quiet or without extraneous acoustic cues correlated with direction and velocity of source movement has been challenging (Perrott, Saberi, Brown, \& Strybel, 1990). Another difficulty, which has become more significant in recent years, is the problem of generating and presenting spatially dynamic auditory stimuli in fMRI neuroimaging studies in which use of real-moving sound sources is impractical (Smith et al., 2004). In addition to these problems, a major complication involves the complexity of simulating motion of wideband, filtered, or natural (e.g., speech or music) waveforms in real time. This is particularly true for motion based on dynamic changes in interaural delay, a major cue to sound localization along the azimuth (Hafter, 1984; Rayleigh, 1907; Yost \& Hafter, 1987). In previous studies, a variety of signal-processing techniques have been used to simulate interaural delay-based motion. Among these are binaural beat waveforms that use pure tones of slightly different frequencies presented to the two ears (Perrott \& Nelson, 1969; Saberi, Tirtabudi, Petrosyan, Perrott, \& Strybel, 2002, 2003), FM noises that produce nonlinear (sinusoidal) motion (Grantham \& Wightman, 1978), and summing time-domain-generated sinusoids combined with a binaural beat paradigm to simulate motion of broadband sounds (Smith et al., 2004). The latter technique, although successful in generating linear motion, ${ }^{1}$ is extremely timeconsuming for standard stimulus sampling rates and durations (e.g., $0.1-1 \mathrm{sec}$ at $44.1 \mathrm{kHz}$ ) and would require calculation of hundreds of sinusoids with independent amplitudes and phases. This would necessitate the generation and storage of numerous independent samples of such waveforms prior to an experimental run, since generating even a single sample of such stimuli between trials is impractical. A further limitation of these techniques is that they generally cannot be applied to nonstationary waveforms, such as speech or other natural sounds. 


\section{Spectral Component Spacing in Complementary Discrete Fourier Transforms}

A novel DSP technique is described here that allows calculation of broadband or filtered waveforms between trials, eliminating the need for storage or computational delays. The technique allows generation of a motion stimulus of any bandwidth and motion velocity based on a linear shift in interaural delay.

A discrete time-domain waveform, $x(n), n=0,1, \ldots$, $N-1$, may be represented in the frequency domain by its discrete Fourier transform (DFT):

$$
X(k \Delta \omega)=\frac{1}{N} \sum_{n=0}^{N-1} x(n) e^{-j \frac{2 \pi n k}{N}} ; k=0,1, \ldots, N-1,
$$

where

$$
\Delta \omega=\frac{\Omega}{N} .
$$

$N$ is the number of samples, $\Omega$ is the sampling frequency, and $\Delta \omega$ is the frequency resolution or bin spacing of the DFT output in hertz (i.e., how far apart the samples are in the frequency domain). Note that the frequency components $k \Delta \omega$ are equally spaced starting at 0 (the dc value) and ending at $N-1(\Delta \omega)$. The spacing $\Delta \omega$ depends on $N$, which is directly related to the waveform duration. For a fixed sampling frequency, $\Delta \omega$ is inversely proportional to the waveform duration - that is, stimulus duration in seconds $(T=N / \Omega=1 / \Delta \omega)$. Thus, spectral energy is measured only at the harmonics of $1 / T$.

We use this concept to generate acoustic stimuli at the two ears that simulate a dynamic linear shift in interaural delay (i.e., motion). In its simplest form, the concept utilizes the idea of binaural beats. If the frequency of a pure tone stimulus presented to one ear is $f$ and that of the stimulus to the other ear is $f+\Delta f$, a single auditory image in motion is usually perceived for $\Delta f$ values of less than $3 \mathrm{~Hz}$ as the waveforms at the two ears modulate in relative phase (Licklider, Webster, \& Hedlun, 1950). At greater $\Delta f$ values, motion is replaced by loudness fluctuation, a roughness quality, or two separate images. Motion velocity in this paradigm may be specified as the rate of change in interaural delay, $\mu \mathrm{sec} / \mathrm{sec}$, which depends on $f$ and $\Delta f$ (i.e., for $\Delta f=1 \mathrm{~Hz}$, the interaural delay shifts by one period of $f$ each second). Because a component that is higher in frequency than $f$ has a shorter period, in order for a higher frequency component to maintain the same rate of change in interaural delay as the lower component, $\Delta f$ at the contralateral ear must be proportionately larger and, thus, must have a higher beat frequency. For example, a frequency component of $500 \mathrm{~Hz}$ with a $1-\mathrm{Hz}$ beat frequency $(500 \mathrm{~Hz}$ to one ear and $501 \mathrm{~Hz}$ to the other ear) simulates a motion velocity of $2,000 \mu \mathrm{sec} / \mathrm{sec}$. In order for a $1000-\mathrm{Hz}$ component to generate a dynamic shift in interaural delay of the same velocity, it must be matched at the contralateral ear by a beat frequency of $2 \mathrm{~Hz}$ ( 1000 and $1002 \mathrm{~Hz}$, respectively).
The DFT components of a complex waveform are separated by the inverse of the waveform duration - that is, $\Delta \omega=1 / T \mathrm{~Hz}$. For a stimulus duration of $100 \mathrm{msec}$, this spacing is $10 \mathrm{~Hz}$. A $100-\mathrm{msec}$ broadband waveform will thus have energy at $\{0, \ldots, 500,510, \ldots, 1000,1010$, ... $\mathrm{Hz}$, effectively up to the Nyquist limit. Simulation of linear motion would require component frequencies at one ear that increase proportionately relative to the corresponding component at the other ear. In the present example, a velocity of $2,000 \mu \mathrm{sec} / \mathrm{sec}$ would require component frequencies to the contralateral ear equal to $\{0, \ldots$, $501,511.02, \ldots, 1002,1012.02, \ldots\} \mathrm{Hz}$. To generate such a dichotic waveform, one simply has to select the appropriate component spacing, which in this case is $\Delta \omega_{2}=$ $\Delta \omega_{1}(1+V)=10.02 \mathrm{~Hz}$, where $V$ is velocity in sec $/ \mathrm{sec}$. A time-domain array may then be generated, representing the contralateral waveform with a duration of $T_{2}=\Delta \omega_{2}{ }^{-1}$ $=\left[\Delta \omega_{1}(1+V)\right]^{-1}$. This shorter duration waveform $\left(N_{2}=\right.$ $\Omega T_{2}$ points) will result in an appropriately wider component spacing and create exactly the type of matched components at the left and right ears that simulate the desired linear change in interaural delay. This array's bins are then filled with the matched frequency components (amplitude and phase spectrum) of the waveform to Channel $1 \mathrm{ob}-$ tained from its DFT:

$$
X_{2}\left(k \Delta \omega_{2}\right)=X_{I}\left(k \Delta \omega_{1}\right) \text { for } k=0,1, \ldots, N_{2}-1 \text {, }
$$

with nonmatching bins (due to the different number of samples) zeroed out. ${ }^{2}$ Inverse Fourier transform of $X_{2}$ will then provide the time-domain signal for this channel.

\section{Sample Waveforms}

An example is given here of how complementary DFTs may be generated for a typical auditory motion experiment. Waveform parameters for this example include a duration of $0.1 \mathrm{sec}$ for Channel 1 (e.g., the left ear), a sampling frequency of $44.1 \mathrm{kHz}$, a velocity of $2,000 \mu \mathrm{sec} / \mathrm{sec}$, and a "distance" traveled of $100 \mu \mathrm{sec}$ starting at an interaural delay of $0 \mu \mathrm{sec}$ (i.e., simulating a starting point directly in front of an observer). Assuming that a $100-\mathrm{msec}$ waveform (e.g., Gaussian noise) is generated for Channel 1, the task, then, is to generate the appropriate complimentary waveform for Channel 2 (e.g., the right ear).

As a first step, the DFT component spacing for Channel 2 must be determined. The component spacing for Channel 1 is $10 \mathrm{~Hz}(1 / T)$ - that is, the first frequency component after the dc component is $10 \mathrm{~Hz}$. To produce a velocity of $2,000 \mu \mathrm{sec} / \mathrm{sec}(0.002 \mathrm{sec} / \mathrm{sec})$, the frequency of the first component (after the dc component) for Channel 2 must be $10+\Delta f$, where $\Delta f=V / \tau$ and $\tau$ is the first component's period $(0.1 \mathrm{sec}$ for $10 \mathrm{~Hz})$. This results in $\Delta f=0.02 \mathrm{~Hz}$, a frequency spacing of $\Delta \omega_{2}=10.02 \mathrm{~Hz}$ for Channel 2, and a Channel 2 stimulus duration of $T 2=$ $1 / \Delta \omega_{2}=99.80039920160 \mathrm{msec}$. Given a frequency spacing $\Delta \omega_{2}=10.02 \mathrm{~Hz}$, the $51 \mathrm{st}$ component at Channel 1 will 
be $500 \mathrm{~Hz}$ and at Channel 2 it will be $501 \mathrm{~Hz}$. The 101st component at Channel 1 will be $1000 \mathrm{~Hz}$ and at Channel 2 it will be $1002 \mathrm{~Hz}$ and so forth. ${ }^{3}$

To summarize, one should select a waveform duration for Channel 2 that results in the appropriate DFT component spacing at that channel. Having generated Channel 2's array, one may fill in this array's bins with complex numbers from the corresponding DFT of Channel 1 to generate complementary amplitude and phase spectra at Channel 2. Since the two channels have different numbers of points (fewer points for the shorter duration waveform), one must zero out the remaining bins of Channel 1 that do not have complementary bins at Channel 2 . This procedure will thus produce exactly the same amplitude and phase spectra at the two ears, with the exception that each component of Channel 2 will be marginally shifted in frequency to simulate motion based on a linear shift in interaural delay. To generate motion that has a starting point other than zero (i.e., directly ahead), the components of one channel may be shifted in phase (linear phase-byfrequency shift; see the Appendix). To generate bandpass motion stimuli, one simply has to zero out those components above and below the passband. Figure 1 shows a sample noise waveform generated using this procedure. Note that Channel 2 contains a noise waveform that progressively shifts in position relative to Channel 1 . We have generated and used sample broadband and band-limited audio waveforms using this procedure. These stimuli produce percepts of smooth intracranial motion of a punctate auditory image (see Archived Materials).

Finally, we should caution that the selection of appropriate motion parameters is critically important to generating a strong percept of motion. The starting interaural delay usually should not exceed $800 \mu \mathrm{sec}$ if one intends to produce motion within the natural range of interaural delays. This value corresponds to a sound source positioned opposite one ear (displaced horizontally by $90^{\circ}$ from di-

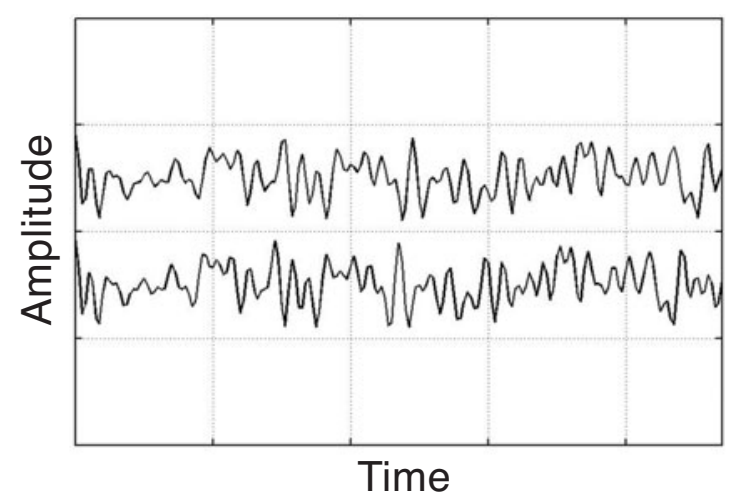

Figure 1. Gaussian noise waveforms generated using the complementary discrete Fourier transform algorithm. The two waveforms start in phase and gradually drift apart, simulating motion based on a dynamic change in interaural delay. rectly in front of the listener). Nonetheless, the technique described here does allow the selection of an arbitrarily large starting or ending interaural delay, which, although unnatural, may be of use in investigating the limits of binaural processing (Saberi, Tirtabudi, et al., 2002). Furthermore, although this DSP technique can generate motion of very high velocity, it is not likely that stimuli with velocities greater than several thousand $\mu \mathrm{sec} / \mathrm{sec}$ (several hundred angular degrees per second) will be perceived as moving (Saberi, Takahashi, et al., 2002).

\section{Summary and Conclusion}

A DSP technique has been described in which complementary DFTs with slightly different frequency component spacings are used for rapid generation of auditory motion stimuli based on a dynamic change in interaural delay. The advantage of the technique is that it eliminates the need for storage of large numbers of complex stimuli prior to an experimental run, and that it is able to generate motion of any velocity for any type of complex sound, including nonstationary waveforms such as speech.

\section{REFERENCES}

Ahissar, M., Ahissar, E., Bergman, H., \& VaAdia, E. (1992). Encoding of sound-source location and movement: Activity of single neurons and interactions between adjacent neurons in the monkey auditory cortex. Journal of Neurophysiology, 67, 203-215.

Altman, J. A., \& Viskov, D. V. (1977). Discrimination of perceived movement velocity for fused auditory image in dichotic stimulation. Journal of the Acoustical Society of America, 61, 816-819.

Blauert, J. (1972). On the lag of lateralization caused by interaural time and intensity differences. Audiology, 11, 265-270.

Chandler, D. W., \& Grantham, D. W. (1992). Minimum audible movement angle in the horizontal plane as a function of stimulus frequency and bandwidth, source azimuth, and velocity. Journal of the Acoustical Society of America, 91, 1624-1636.

Dove, H. W. (1839). Nachtrag zu den Combinationstonen. In Repertorium der Physik (pp. 404-405). Berlin: Verlag von Veit.

Grantham, D. W. (1984). Discrimination and dynamic interaural intensity differences. Journal of the Acoustical Society of America, 76, 71-76.

Grantham, D. W. (1986). Detection and discrimination of simulated motion of auditory targets in the horizontal plane. Journal of the Acoustical Society of America, 79, 1939-1949.

Grantham, D. W., \& Wightman, F. L. (1978). Detectability of varying interaural temporal differences. Journal of the Acoustical Society of America, 63, 511-523.

HAFTER, E. R. (1984). Spatial hearing and the duplex theory: How viable is the model? In. G. M. Edelman, W. E. Gall, \& W. M. Cowan (Eds.) Dynamic aspects of neocortical function (pp. 425-448). New York: Wiley.

Licklider, J. C. R., Webster, J. C., \& Hedlun, J. M. (1950). On the frequency limits of binaural beats. Journal of the Acoustical Society of America, 22, 468-473.

Moiseff, A., \& HAREsign, T. (1992). Response of auditory units in the barn owl's inferior colliculus to continuously varying interaural phase differences. Journal of Neurophysiology, 67, 1428-1436.

Pavani, F., Macaluso, E., Warren, J. D., Driver, J., \& Griffiths, T. D. (2002). A common cortical substrate activated by horizontal and vertical sound movement in the human brain. Current Biology, 12, 1584-1590.

Perrott, D. R., \& Musicant, A. D. (1977a). Minimum auditory movement angle: Binaural localization of moving sound sources. Journal of the Acoustical Society of America, 62, 1463-1466. 
Perrott, D. R., \& Musicant, A. D. (1977b). Rotating tones and binaural beats. Journal of the Acoustical Society of America, 61, 1288-1292.

Perrott, D. R., \& Nelson, M. A. (1969). Limits for the detection of binaural beats. Journal of the Acoustical Society of America, 46, 1477-1481.

Perrott, D. R., Saberi, K., Brown, K., \& Strybel, T. Z. (1990). Auditory psychomotor coordination and visual search performance. Perception \& Psychophysics, 48, 214-226.

Perrott, D. R., \& Tucker, J. (1988). Minimum audible movement angle as a function of signal frequency and the velocity of the source. Journal of the Acoustical Society of America, 83, 1522-1527.

Peterson, J. (1916). The nature and probable origin of binaural beats. Psychological Review, 23, 333-351.

Rauschecker, J. P., \& HARRIS, L. R. (1989). Auditory and visual neurons in the cat's superior colliculus selective for the direction of apparent motion stimuli. Brain Research, 490, 56-63.

RAYLEIGH, LORD (1876). Our perception of the direction of a sound source. Nature, 14, 32-33.

RAYleigh, LORD (1907). On our perception of sound direction. Philosophical Magazine, 13 (Series 6), 214-232.

SABERI, K. (1996). An auditory illusion predicted from a weighted crosscorrelation model of binaural interaction. Psychological Review, 103 $137-142$.

Saberi, K., \& Perrott, D. R. (1990). Minimum audible movement angles as a function of sound-source trajectory. Journal of the Acoustical Society of America, 88, 2639-2644.

Saberi, K., Takahashi, Y., Egnor, R., Farahbod, H., Mazer, J., \& KonISHI, M. (2002). Detection of large interaural delays and its implication for models of binaural interaction. Journal of the Association for Research in Otolaryngology, 3, 80-88.

Saberi, K., Tirtabudi, P., Petrosyan, A., Perrott, D. R., \& StryBEL, T. Z. (2002). Concurrent motion detection based on dynamic changes in interaural delay. Hearing Research, 174, 149-157.

Saberi, K., Tirtabudi, P., Petrosyan, A., Perrott, D. R., \& StryBEL, T. Z. (2003). Detection of dynamic changes in interaural delay. Acta Acustica United With Acustica, 89, 333-338.

Smith, K. R., OKadA, K., SABeri, K., \& HickoK, G. (2004). Human cortical auditory motion areas are not motion selective. NeuroReport, 15, 1523-1526.

Spitzer, M. W., \& Semple, M. N. (1991). Interaural phase coding in auditory midbrain: Influence of dynamic stimulus features. Science, 254, 721-723.

Strybel, T. Z., \& Fujimoto, K. (2000). Minimum audible angles in the horizontal and vertical planes: Effects of stimulus onset asynchrony and burst duration. Journal of the Acoustical Society of America, 108, 3092-3095.

Strybel, T. Z., Manligas, C. L., \& Perrott, D. R. (1989). Auditory apparent motion under binaural and monaural listening conditions. Perception \& Psychophysics, 45, 371-377.

Strybel, T. Z., Span, S. A., \& Witty, A. M. (1998). The effect of timing and spatial separation on the velocity of auditory apparent motion. Perception \& Psychophysics, 60, 1441-1451.

Stumpf, E., Toronchuk, J. M., \& CynAdER, M. S. (1992). Neurons in cat primary auditory cortex sensitive to correlates of auditory motion in three-dimensional space. Experimental Brain Research, 88, 158-168.

Thompson, S. P. (1877). On binaural audition. Philosophical Magazine, 4 (Series 5), 274-276.

Thompson, S. P. (1878). Phenomena of binaural audition. Philosophical Magazine, 6 (Series 5), 383-390.
Valentine, W. L. (1928). Notes on the binaural beat. Comparative Psychology, 7, 357-368.

Warren, J. D., Zielinski, B. A., Green, G. G. R., Rauschecker, J. P., \& Griffiths, T. D. (2002). Perception of sound-source motion by the human brain. Neuron, 34, 139-148.

Waugh, W., Strybel, T. Z., \& Perrott, D. R. (1979). Perception of moving sounds: Velocity discrimination. Journal of Auditory Research, 19, 103-110.

Yost, W. A., \& Hafter, E. R. (1987). Lateralization. In W. A. Yost \& G. Gourevitch (Eds.), Directional hearing (pp. 49-84). New York: Springer-Verlag.

\section{NOTES}

1. Here, linear motion refers to a linear change in interaural delay approximating a constant velocity source at a fixed distance from the observer.

2. For this example, the highest frequency components of the original waveform will be eliminated. However, given a typical sampling frequency of $44.1 \mathrm{kHz}$, the eliminated components are above the highest frequency audible to humans $(20 \mathrm{kHz})$ and, thus, inconsequential. To retain all points from the original waveform, one may set $\Delta \omega_{2}$ smaller than $\Delta \omega_{1}$, resulting in an array with a greater number of points than the original waveform [i.e., $\left.\Delta \omega_{2}=\Delta \omega_{1}(1-V)\right]$. The effect is simply a change in the direction of motion (see the Appendix).

3 . The duration is rounded to the nearest digital sampling period, which, for a sampling rate of $44.1 \mathrm{kHz}$, is $\sim 23 \mu \mathrm{sec}$. Thus, the maximum quantization error in estimating duration is $11.5 \mu \mathrm{sec}$, which, for a $1-\mathrm{sec}$ stimulus, results in a maximum frequency shift of less than $0.00002 \mathrm{~Hz}$.

\section{ARCHIVED MATERIALS}

The following materials associated with this article may be accessed through the Psychonomic Society's Norms, Stimuli, and Data archive, http://www.psychonomic.org/archive/.

To access these files, search the archive for this article using the journal (Behavior Research Methods, Instruments, \& Computers), the author's name (Saberi), and the publication year (2004).

FILE: Saberi-BRMIC-2004.zip.

DESCRIPTION: The compressed archive file contains five files:

stationary_noise.wav, an audio file of spatially stationary burst of Gaussian noise.

stationary_speech.wav, an audio file of spatially stationary recorded speech.

noise_in_motion.wav, an audio file of spatially moving Gaussian noise, with a starting interaural delay of $800 \mu$ sec leading to one ear and an ending interaural delay of at $800 \mu \mathrm{sec}$ leading to the contralateral ear, with a motion velocity of $800 \mu \mathrm{sec} / \mathrm{sec}$.

speech_in_motion.wav, an audio file of spatially moving recorded speech with a velocity of $500 \mu \mathrm{sec} / \mathrm{sec}$.

motion.m, an executable Matlab program that loads any mono file (labeled monostimulus.wav) and transforms it into a motion stimulus (labeled motionstimulus.wav). Requires the basic version of the Matlab programming application (a signal-processing toolbox is not needed). All sample waveforms were recorded at a rate of $44.1 \mathrm{kHz}$. Requires listening through stereo headphones.

AUTHOR's E-MAIL ADDRESS: saberi@uci.edu.

AUTHOR's WEB SITE: http://orion.oac.uci.edu/ kourosh/. 
APPENDIX

Matlab Program for Generating Stereo Motion From Any Mono Stimulus

\begin{tabular}{|c|c|}
\hline clear; & \\
\hline$\% * * * * * * * * * * * * * * * * * * * * * * * * * * * * * * * * *$ & Stimulus parameters $* * * * * * * * * * * * * * * * * * * * * * * *$ \\
\hline$[\mathrm{y}, \mathrm{fs}]=$ wavread('monostimulus'); & \%load mono wav file ( $\mathrm{fs}=$ sampling frequency) \\
\hline ly=length $(y)$ & $\%$ Number of samples in $y$ \\
\hline dur $1=(1 y-1) / f s$ & $\%$ Duration of channel 1 waveform (in seconds) \\
\hline $\mathrm{t}=0: 1 / \mathrm{fs}: \mathrm{dur} 1$ & $\%$ Time vector \\
\hline$v=250$ & $\%$ Velocity in microseconds/seconds \\
\hline $\mathrm{v}=\mathrm{v} . / 1 \mathrm{e} 6$ & $\%$ Convert to $\mathrm{s} / \mathrm{s}$ \\
\hline $\mathrm{id}=0$ & $\%$ Starting value of interaural delay in microseconds \\
\hline$\%$ & Usually takes value between -800 to 800 \\
\hline$\% * * * * * * * * * * * * * * * * * * * * * * * * * * * * * * * * *$ & Create Channel 2 waveform ****************** \\
\hline if $\min (\operatorname{size}(y))==2 ; y(:, 2)=[] ;$ end; & $\%$ Delete channel 2 if uploaded waveform is not mono \\
\hline Dw $1=1 /$ dur 1 & $\%$ Frequency component spacing for channel 1 \\
\hline Dw2=Dw1*(1-v); & $\%$ Frequency component spacing for channel 2 \\
\hline dur2=1/Dw2; & $\%$ Duration of waveform to channel 2 \\
\hline hzl=(0:1/dur1:fs)'; & $\%$ Array of Channel 1 component frequencies \\
\hline $\mathrm{hz} 2=(0: 1 / \text { dur2:fs })^{\prime}$ & $\%$ Array of Channel 2 component frequencies \\
\hline lhzl=length(hz1); & $\%$ Length of Array 1 \\
\hline lhz2=length $(\mathrm{hz} 2)$ & $\%$ Length of Array 2 \\
\hline$x(\operatorname{lhz} 2,2)=0$ & $\%$ Create zero array based on longer stimulus \\
\hline$f y=f f t(y)$ & $\%$ Fast Fourier Transform of Channel 1 waveform \\
\hline $\begin{array}{l}\text { lyy }=\text { floor }(1 y / 2)+1 ; \\
\text { fy } 2=\text { hz } 2 * 0 ;\end{array}$ & \\
\hline fy $2(1:$ lyy $)=f y(1: l y y)$ & \%Fill Channel 2 with Channel 1 components \\
\hline fy $2(\operatorname{lhz} 2-l y y+1: \operatorname{lnz} 2)=$ fy $($ ly-lyy $+1: l y)$ & \\
\hline $\mathrm{x}(:, 2)=\operatorname{real}(\mathrm{ifft}(\mathrm{fy} 2))$ & \%Transform Channel 2 into time domain \\
\hline $\mathrm{x}(1: \operatorname{ly}, 1)=\mathrm{y}$ & \%Channel 1 waveform \\
\hline p1=angle (fy)-hz1 $*^{*} 2 * \mathrm{pi}^{*}(\mathrm{id} / 1 \mathrm{e} 6)$; & \%Linear phase shift (starting interaural delay) \\
\hline $\mathrm{r} 1=\mathrm{abs}(\mathrm{fy})$ & \%Magnitude spectrum \\
\hline $\mathrm{z} 1=\mathrm{r} 1 . * \exp (\mathrm{i} \cdot * \mathrm{p} 1)$ & $\%$ New complex numbers for waveform 2 \\
\hline $\mathrm{x} 1=\operatorname{real}(\mathrm{ifft}(\mathrm{z} 1))$ & \%Transform Channel 2 to time domain waveform \\
\hline $\mathrm{x}(1: \mathrm{ly}, 1)=\mathrm{x} 1$ & $\%$ Channel 1 time waveform \\
\hline wavwrite(x,fs,'motionstimulus'); & $\%$ write the final waveform to a .wav file \\
\hline
\end{tabular}

(Manuscript received February 11, 2004; revision accepted for publication July 17, 2004.) 